\title{
A Generalisation of a Theorem of E. Toeplitz
}

\author{
By J. Anderson, St Andrews University. \\ (Received 13th April, 1933. Read 5th May, 1933.)
}

§. Introduction.

In the Mathematische Annalen, 11 (1877), 440-444, E. Toeplitz has proved the following theorem: If three quadrics are polar quadrics of three points with respect to a cubic surface, then their $(2,2,2)$ invariant vanishes; the invariant being of the second degree in the coefficients of each of the three quadrics.

The original proof, as given by Toeplitz, is complicated, but its underlying principles are revealed very clearly by combining the notation of the symbolic invariant theory with that of matrices. This brings out at once the interesting fact that the property is general, and applies to quadrics and cubics in space of, not merely three, but any odd number of dimensions.

\section{§2. Notation.}

Let the non-symbolic forms of three quadrics in a space of $m-1$ dimensions be $f_{1}=\Sigma a_{i j} x_{i} x_{j}, f_{2}=\Sigma b_{i j} x_{i} x_{j}$, and $f_{3}=\Sigma c_{i j} x_{i} x_{j}$, where $i, j=1,2, \ldots, m$.

Symbolically these are

$$
\begin{aligned}
& f_{1} \equiv a_{x}^{2}=a_{x}^{\prime 2}=a_{x}^{\prime 2}=\ldots=\left(a_{1} x_{1}+a_{2} x_{2}+\ldots+a_{m} x_{m}\right)^{2}, \\
& f_{2} \equiv b_{x}^{2}=b_{x}^{\prime 2}=b_{x}^{\prime \prime 2}=\ldots \ldots \text {, } \\
& f_{3} \equiv c_{x}^{2}=c_{x}^{\prime 2}=c^{\prime \prime 2}{ }_{x}=\ldots \text {, } \\
& \text { where } a_{i} a_{j}=a_{i j}=a_{j} a_{i}=a_{j i} \text {, etc. }
\end{aligned}
$$

\section{\$3. Statement of the theorem.}

The necessary and sufficient condition that the three quadrics, $a_{x}^{2}, b_{x}^{2}, c_{x}^{2}$, in the $(m-1)$ manifold be the polar quadrics of three points $\xi, \eta, \zeta$ with respect to a cubic primal $t_{x}^{3}$ is that

where (1) $m=2 n$,

$$
\left(A_{n} B_{n}\right)\left(B_{n} C_{n}\right)\left(C_{n} A_{n}\right)=0
$$

and (2) $A_{n}=a a^{\prime} a^{\prime \prime} \ldots a^{(n-1)}$, a convolution of $n$ equivalent symbols $a$. 


\section{§4. Proof.}

The polar quadric of a point $\xi=\left\{\xi_{1}, \xi_{2}, \ldots, \xi_{m}\right\}$ with respect to $t_{x}^{3}$ is $t_{x}^{2} t_{\xi}$, and this is to be the same quadric as $a_{x}^{2}$. Hence $t_{x}^{2} t_{\xi}=\pi a_{x}^{2}$. Similarly, $t_{x}^{2} t_{\eta}=\rho b_{x}^{2}$ and $t_{x}^{2} t_{\zeta}=\chi c_{x}^{2}$, where $\pi, \rho, \chi$ are scalar.

Polarising these three equations with respect to an arbitrary point $y$, we get the following relations:

$$
t_{x} t_{y} t_{\xi}=\pi a_{x} a_{y} ; \quad t_{x} t_{y} t_{\eta}=\rho b_{x} b_{y} ; \quad t_{x} t_{y} t_{\zeta}=\chi c_{x} c_{y}
$$

whence we see that

$$
\rho b_{x} b_{\zeta}=\chi c_{x} c_{\eta} ; \quad \chi c_{x} c_{\xi}=\pi a_{x} a_{\zeta} ; \quad \pi a_{x} a_{\eta}=\rho b_{x} b_{\xi} .
$$

In matrix notation, the first of these becomes $\rho x^{\prime} Q \zeta=\chi x^{\prime} R \eta$, where the accent attached to $x$ denotes transposition; that is, if $x$ is a column, then $x^{\prime}$ is a row vector. This is indicated by the kind of brackets we use:

$$
\begin{aligned}
& x^{\prime}=\left[x_{1}, x_{2}, \ldots, x_{m}\right], \\
& \zeta=\left\{\zeta_{1}, \zeta_{2}, \ldots, \ldots, \zeta_{m}\right\} . \\
& \text { Again, } \quad Q=\left(b_{i j}\right)=\left[\begin{array}{ccccc}
b_{11} & b_{12} & b_{13} & \ldots & b_{1 m} \\
b_{21} & b_{22} & \cdot & & \cdot \\
b_{31} & \cdot & \cdot & & \cdot \\
\cdot & & & \\
b_{m 1} & . & . & . & b_{m m}
\end{array}\right] .
\end{aligned}
$$

Hence we have the following equations:

$$
\begin{aligned}
& x^{\prime}\left(Q \frac{\zeta}{\chi}-R \frac{\eta}{\rho}\right)=0, \\
& x^{\prime}\left(R \frac{\xi}{\pi}-P \frac{\zeta}{\chi}\right)=0, \\
& x^{\prime}\left(P \frac{\eta}{\rho}-Q \frac{\xi}{\pi}\right)=0 .
\end{aligned}
$$

Eliminating the $3 m$ scalar quantities $\frac{\xi_{i}}{\pi}, \frac{\eta_{i}}{\rho}, \frac{\zeta_{i}}{\chi},(i=1,2, \ldots, m)$, from the equations (2), (3), (4), we get a vanishing determinant of $3 \mathrm{~m}$ rows and columns, conveniently represented as follows:

$$
|X|=\left|\begin{array}{rrr}
0 & -R & Q \\
R & 0 & -P \\
-Q & P & 0
\end{array}\right|=0 .
$$


We shall shew that this skew-symmetric determinant, when expanded and expressed in symbolic form, is equal to

$$
\left(\frac{1}{n !}\right)^{6} \cdot\left(\left(A_{n} B_{n}\right)\left(B_{n} C_{n}\right)\left(C_{n} A_{n}\right)\right)^{2}
$$

Conversely, if this invariant vanishes, the argument can be reversed; we should get consistent equations (1), giving possible values for the coefficients of the cubic form.

Pre-multiplying the matrix $X$ of the above equations by $K H$ where

$$
\text { we have } \quad H=\left[\begin{array}{ccc}
1 & Q P^{-1} & 0 \\
0 & 1 & 0 \\
0 & 0 & 1
\end{array}\right], \quad K=\left[\begin{array}{ccc}
1 & 0 & R P^{-1} \\
0 & 1 & 0 \\
0 & 0 & 1
\end{array}\right] \text {, }
$$

But $K, H$ are evidently unimodular matrices; that is, $|K|=|H|=1$.

Hence

$$
|X|=\left|Q P^{-1} R-R P^{-1} Q\right| \cdot|P|^{2} .
$$

The sign in front of the determinant is settled by the fact that the matrix $P$ is of even order $2 n$. Again, the matrix $S=Q P^{-1} R-R P^{-1} Q$ is skew symmetric and of even order: hence its determinant is a perfect square.

Further, we can easily shew that $|S|$ is an invariant of the three quadrics.

Let the matrix equation $x=M \bar{x}$ denote a linear transformation from the variables $x$ to the new variables $\bar{x}$; and let $x^{\prime} P x=\bar{x}^{\prime} \bar{P} \bar{x}$. Hence $\bar{x}^{\prime} M^{\prime} P M \bar{x}=\bar{x}^{\prime} \bar{P} \bar{x}$ : or $M^{\prime} P M=\bar{P}$. Similarly for $Q$ and $R$.

Therefore $\bar{Q} \bar{P}^{-1} \bar{R}=\left(M^{\prime} Q M\right)\left(M^{-1} P^{-1} M^{\prime-1}\right)\left(M^{\prime} R M\right)$

$$
=M^{\prime} Q P^{-1} R M \text {. }
$$

Hence

$$
\begin{aligned}
\overline{\mathcal{S}} & =\bar{Q} \bar{P}^{-1} \bar{R}-\bar{R} \bar{P}^{-1} \bar{Q} \\
& =M^{\prime}\left(Q P^{-1} R-R P^{-1} Q\right) M \\
& =M^{\prime} S M:
\end{aligned}
$$

or

$$
|\bar{S}|=|S| \cdot|M|^{2} \text {. }
$$


The transformation also replaces $|P|$ by $|P| \cdot|M|^{2}$, and hence replaces $|X|$ by $\left.|S||M|^{2} \cdot P\right|^{2}|M|^{4}$; that is

$$
|\bar{X}|=\left.! S|| P\right|^{2}|M|^{6} \text {, }
$$

which shews that $|X|$ is an invariant of weight six. Its square root is an invariant of weight three and may be calculated from a convenient canonical form.

Since any two general quadrics can be reduced to forms giving matrices of the unit and diagonal types, let us leave $R$ general, and take $P$ as the unit matrix $I$, and $Q$ as the diagonal matrix $\operatorname{diag}\left(b_{1}, b_{2}, b_{3}, \ldots, b_{m}\right)$. Hence we get simply for $(5),|X|=|Q R-R Q|$. But in this case,

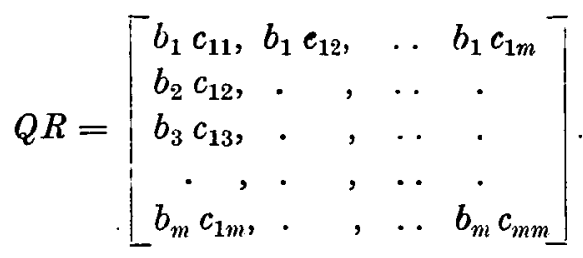

Hence $\quad|X|=\left|c_{i j}\left(b_{i}-b_{j}\right)\right|$, where $i, j=1,2, \ldots, m$.

We shall assume the lemma (proved hereafter in $\S 6$ ) that, when this determinant is expanded and its terms re-arranged, we get

or

$$
\sqrt{ }|X|=(-)^{n / 2} \Sigma b_{i} b_{j} \ldots b_{k}\left(C_{i j \ldots k}\right)_{r \delta \ldots t}
$$

according as $n=\frac{1}{2} m$ is even or odd. The following properties are to be noted.

(i) There are $\frac{m !}{n ! n !}$ terms in each summation.

(ii) For our present purpose, the sign in front of $\Sigma$ is of no importance, and we shall write $\sqrt{ }|X|= \pm \sum b_{i} b_{j} \ldots b_{k}\left(C_{i j \ldots k}\right)_{r \varepsilon \ldots t}$.

(iii) Again, $(i j \ldots k)$ and $(r s \ldots t)$ are each sequences of $n$ letters and are algebraic complements for the sequence $(123 \ldots m)$.

(iv) The sign convention is determinantal; that is, $(i j \ldots . . k r s \ldots t)$ is regarded as positive, whereas $(j i \ldots k r s . \ldots t)$, for example. is negative. 
a Generalisation of a Theorem of E. Toeplitz

(v)

$$
\left(C_{i j \ldots k}\right)_{r \varepsilon \ldots t}=\left|\begin{array}{cccc}
c_{i r} & c_{i s} & \ldots & c_{i t} \\
c_{j r} & \cdot & & \cdot \\
\cdot & \cdot & & \cdot \\
c_{k r} & \cdot & \ldots & c_{k t}
\end{array}\right| .
$$

Symbolically then

$$
\begin{aligned}
\left(C_{i j \ldots k}\right)_{r s \ldots t} & =\frac{1}{n !}\left(c c^{\prime} c^{\prime \prime} \ldots c^{(n-1)}\right)_{i j \ldots k}\left(c c^{\prime} c^{\prime \prime} \ldots c^{(n-1)}\right)_{r s \ldots t} \\
& =\frac{1}{n !} C_{i j \ldots k} C_{r \varepsilon \ldots t},
\end{aligned}
$$

and the symbol for the product $b_{i} b_{j} \ldots b_{k}$ is $-\frac{1}{n !} B_{i j \ldots k}^{2}$, similarly.

Hence

$$
(n !)^{2} \sqrt{ }|X|= \pm \Sigma B_{i j \ldots k}^{2} C_{i j \ldots k} C_{r s \ldots t} .
$$

Now

$$
\left(A_{n} B_{n}\right)\left(B_{n} C_{n}\right)\left(C_{n} A_{n}\right)=\Sigma A_{i j \ldots k} B_{r s \ldots t} \Sigma B_{i j \ldots k} C_{r s \ldots \ell} \Sigma C_{i j \ldots k} A_{r \varepsilon \ldots \ell} .
$$

But for the case we have taken, where $A_{n}$ is derived from the unit, and $B_{n}$ from a diagonal, matrix, we have

$$
\begin{aligned}
\text { (i) } A_{i j \ldots k} A_{r s \ldots t}=0 \text { unless }(i j \ldots k)=(r s \ldots t) \text {; } \\
\text { (ii) } A_{i j \ldots k}^{2}=n ! ; \\
\text { (iii) } B_{i j \ldots k} B_{r s, \ldots t}=0 \text { unless }(i j \ldots k)=(r s \ldots t) .
\end{aligned}
$$

Hence

$$
\left(A_{n} B_{n}\right)\left(B_{n} C_{n}\right)\left(C_{n} A_{n}\right)=\Sigma B_{i j \ldots k}^{2} C_{i j \ldots k} C_{r s \ldots i t} .
$$

Comparing this result with (6), we see that

or

$$
\begin{aligned}
\sqrt{ }|X| & = \pm\left(\frac{1}{n !}\right)^{3}\left(A_{n} B_{n}\right)\left(B_{n} C_{n}\right)\left(C_{n} A_{n}\right) \\
|X| & =\left(\frac{1}{n !}\right)^{6} \cdot\left(\left(A_{n} B_{n}\right)\left(B_{n} C_{n}\right)\left(C_{n} A_{n}\right)\right)^{2} .
\end{aligned}
$$

§. Special Cases.

(i) Binary Case, $m=2$.

The binary invariant $(a b)(b c)(c a)$ vanishes if $a_{x}^{2}, b_{x}^{2}, c_{x}^{2}$ (that is, three point-pairs on a line), are first polars of three points with respect to a binary cubic, $t_{x}^{3}$ (which, of course, represents three points 
on the same line): or, equivalently, if the three pairs of points are in involution.

(ii) Quaternary Case, $m=4$.

In this case we get for (7),

$$
|X|=\frac{1}{64}((A B)(B C)(C A))^{2}
$$

which is the result given by Toeplitz.

(iii) $m=2 n-1$.

Since $|X|=\left|c_{i j}\left(b_{i}-b_{j}\right)\right|$, that is, is a skew symmetric determinant, which vanishes if $m$ is odd, there is no definite result in the $(2 n-1)$-ary case.

\section{§6. Proof of Lemma.}

Let $|X|=\left|c_{p q}\left(b_{p}-b_{q}\right)\right|=\left|a_{p q}\right|$, where $p, q=1,2, \ldots, m$. Then we require to shew that

or

$$
\begin{aligned}
\sqrt{ }|X| & =(-)^{n / 2} \Sigma b_{i} b_{j} \ldots b_{k}\left(C_{i j \ldots k}\right)_{r s \ldots t}, \\
& (-)^{(n-1) / 2} \Sigma b_{i} b_{j} \ldots b_{k}\left(C_{i j \ldots k}\right)_{r s \ldots t},
\end{aligned}
$$

according as $n$ is even or odd. The summation sign and the sign conventions have already been explained.

Now $\sqrt{ }|X|$ is a Pfaffian; and Pfaffians of order $m$ can be calculated from those of order $m-2$ in the following way. ${ }^{1}$

If $\sqrt{ }|X|=[1,2, \ldots, m]=\sqrt{ }\left|a_{p q}\right|=\sqrt{ }\left|c_{p q}\left(b_{p}-b_{q}\right)\right|$, then (8), $[1,2, \ldots, m]=a_{12}[3,4, \ldots, m]+a_{13}[4, \ldots, m, 2]+\quad+a_{1 m}[2,3, \ldots, m-1]$ where, after the suffix 1 has been selected, the others follow cyclically.

Again,

$$
[1,2]=a_{12}
$$

and so on.

$$
[1,2,3,4]=a_{12} a_{34}+a_{13} a_{42}+a_{14} a_{23} \text {, }
$$

We shall assume the lemma true for the $m-2$ case; further, we shall take $n-\mathrm{I}$ to be even; that is,

$$
[1,2, \ldots, m-2]=(-)^{(n-1) / 2} \Sigma b_{1} b_{2} \ldots b_{n-1}\left(C_{12 \ldots, n-1}\right)_{n, \ldots, 2 n-2} .
$$

I Scott and Mathews, Theory of Determinants (1904), p. 95. 
But from (8), $[1,2, \ldots, m]$

$$
\begin{aligned}
& =(-)^{(n-1) / 2} c_{12}\left(b_{1}-b_{2}\right) \Sigma b_{3} b_{4} \ldots b_{n+1}\left(C_{34, \ldots, n+1}\right)_{n+2, \ldots, 2 n}+\ldots \\
& +(-)^{(n-1) / 2} c_{1 n}\left(b_{1}-b_{n}\right) \Sigma b_{n+1} b_{n+2} \ldots b_{2 n-1}\left(C_{n+1, \ldots, 2 n-1}\right)_{2 n, 2, \ldots, n-1}+\ldots \\
& +(-)^{(n-1) / 2} c_{1 m}\left(b_{1}-b_{m}\right) \Sigma b_{2} b_{3} \ldots b_{n}\left(C_{23, \ldots, n}\right)_{n+1, \ldots, 2 n-1} .
\end{aligned}
$$

Here the factor $b_{1} b_{2} b_{3} \ldots b_{n}$ occurs in $n$ of the above terms and the sum of the coefficients is easily shewn to be $(-)^{(n-1) / 2}\left(C_{12, \ldots, n}\right)_{n+1, \ldots, 2 n}$ if $n$ is odd. But this is true since we took $n-1$ to be even. Similarly for the other terms, and so we get

$$
[1,2, \ldots, m]=(-)^{(n-1) / 2} \Sigma b_{1} b_{2} b_{3} \ldots b_{n}\left(C_{123 \ldots . n}\right)_{n+1, \ldots, m} \text {. }
$$

The other case, when $n-1$ is odd, is proved in a similar manner.

My thanks are due to Professor Turnbull for supervising this work throughout and freely giving me at all times the benefit of his own wide experience. 